\title{
The Development of Polydimethysiloxane/ZnO-GO Antifouling Coatings
}

\author{
Xiaoxue Zhang ${ }^{1} \cdot$ Erland Årstøl $^{2} \cdot$ Marianne Nymark $^{3} \cdot$ Maxime Fages-Lartaud $^{2} \cdot \varnothing_{\text {yvind Mikkelsen }}{ }^{1}$
}

Received: 26 July 2021 / Accepted: 17 August 2021 / Published online: 31 August 2021

(C) The Author(s) 2021

\begin{abstract}
The development of antifouling coating for sensor is desirable because the biofilm can shorten sensor's life and cause inaccurate reading. In this study, a facile one-pot reaction was used to synthesized $\mathrm{ZnO}$-graphene oxide (GO) ( $\mathrm{ZnO}-\mathrm{GO})$ nanocomposites. Different amount of $\mathrm{ZnO}-\mathrm{GO}$ was incorporated in the polydimethylsiloxane (PDMS) matrix respectively though a simple solution mixing method, in order to create PDMS/ZnO-GO nanocomposite (PZGO). The coating was obtained directly by spin coating of PZGO/tetrahydrofuran suspension. The hydrophobicity, surface roughness (Ra), surface free-energy (SFE) and nanoscale structure were investigated as antifouling factors. Antifouling tests were performed using two marine microorganisms, the cyanobacterium Synechococcus sp. Strain PCC 7002 and the diatom Phaeodactylum tricornutum. PZGO0.2 (mass ratio of ZnO-GO to PDMS: $0.2 \mathrm{wt} \%$ ) displayed excellent antifouling property with $8.5 \%$ of Synechococcus sp. Strain PCC 7002 biofilm coverage, while PZGO0.1 (mass ratio of ZnO-GO to PDMS: $0.1 \mathrm{wt} \%$ ) showed $2.4 \%$ P. tricornutum biofilm coverage. The antifouling property of the synthesized PZGO nanocomposite can be attributed to its high $\mathrm{Ra}$ and hydrophobicity which was caused by the good dispersion of $\mathrm{ZnO}-\mathrm{GO}$ in PDMS matrix. This study suggests a potential of PZGO nanocomposite for sensor's antifouling coating, which could contribute to improve sensor's durability relating to biofouling in future.
\end{abstract}

Graphic Abstract

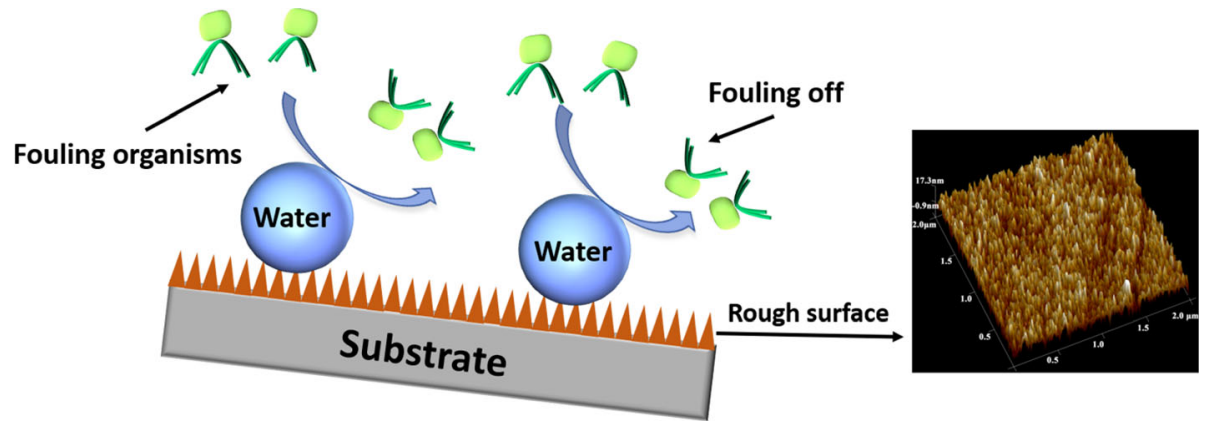

Keywords Polydimethysiloxane (PDMS) - Zinc oxide/graphene oxide (ZnO-GO) · Nanocomposites · Antifouling

Xiaoxue Zhang

xiaoxue206258@gmail.com

1 Department of Chemistry, Norwegian University of Science and Technology, Trondheim, Norway

2 Department of Biotechnology and Food Science, Norwegian University of Science and Technology, Trondheim, Norway

3 Department of Biology, Norwegian University of Science and Technology, Trondheim, Norway

\section{Introduction}

Underwater installations in marine environment are suffering biofouling problems. This also accounts for vessels and warships resulting in increased drag resistance and oil consumption, which in turn results in high $\mathrm{CO}_{2}$ emission and economic loss [1-3]. Besides, a wide range of marine sensors have been set up to monitor seawater properties (e.g. temperature, turbidity, $\mathrm{CO}_{2}$, conductivity...), marine 
ecosystems and environmental contaminants [4-6]. Fouling on these sensors could however disrupt reading and greatly shorten the sensors' life-time [7]. In aquaculture, fouling on cage nets has a negative effect on solidity of the nets as well as on fish growth, health and welfare [8, 9]. The direct economic costs of biofouling control are estimated to $5-10 \%$ of production costs in aquaculture industry (2012 estimate) [8]. In addition, many indirect effects of biofouling remain broadly unassessed, meaning that the overall impact of biofouling in aquaculture is unquestionably underestimated [8]. Meanwhile, it is known that water quality monitoring with sensors is critical in aquaculture since water quality, e.g. $\mathrm{pH}$, dissolved oxygen and carbon dioxide value, is crucial for fish health. The presence of fouling on the surface of sensors causes significant drift, poor signal-to-noise ratio and high background signal, leading to incorrect reading from the sensors $[10,11]$. In consequence, it is urgent to protect the sensors' surface from serious fouling issues.

Antifouling (AF) paints have been widely used to control and prevent biofilm settlement on submerged surfaces. Tributyltin (TBT) based AF paints had been widely used before they were banned by International Maritime Organization (IMO) due to the detrimental impact of TBT on marine environment [12]. Meanwhile, the growing concern for environmental problems has increasingly restricted the use of biocide-based paints. Thus, lots of attention has been attracted to the development of practical and environment friendly silicone-based Fouling Release Coating (FRC) techniques. Polydimethylsiloxane (PDMS) paints possesses nonleachant properties, ultrasmooth topology, environmental friendliness, stability in water, low free energy, optimum molecular mobility, water and fouling repellency, stability against heat and oxidation, and good adhesion on different substrate materials [13]. It can weaken the attachment of fouling organisms due to low surface energy and low elastic modulus, thus promoting the release of accumulated fouling by dynamic forces caused by movement through the water [14]. However, it also suffers from the disadvantage of being less effective on slow moving and stationary installations, such as slow moving or moored vessels, installed sensors, and aquaculture nets and cages [15].

One effective strategy to overcome this weakness is to develop PDMS/nanofillers nanocomposite materials. The nanofillers like metal oxides, noble metals, and graphenebased materials can increase the resistance against microorgram attacks [16]. Nanomaterials like $\mathrm{ZnO}$ nanoparticles (NPs), $\beta-\mathrm{MnO}_{2} \mathrm{Cu}_{2} \mathrm{O}$ nanocubes, titania nanospheres, $\mathrm{Ag} @ \mathrm{SiO}_{2}$, carbon nanofillers (GO, carbon nanotubes (CNT)) have been used as effective nanofillers in antifouling field [17-19]. Sherif A. EI-Safty has made a series of PDMS/nanofiller antifouling coatings, with GO-
$\mathrm{ZnO}$ nanorod, $\mathrm{SiO}_{2}$-doped $\mathrm{ZnO}$ and $\beta-\mathrm{MnO}_{2}$ nanorod working as nanofillers [20-22]. The changed surface topography (nanostructure, surface roughness) and surface wettability leads to the improved fouling release (FR) property and lower adhesion strength between biofilm and surface. Beigbeder et al. have demonstrated that low amounts of CNT can result in a significant enhancement of FR property of PDMS-based coatings [23, 24]. Irani and coworkers showed that the loading of pristine and fluorinated MWCNT into PDMS can reduce the pseudobarnacle adhesion strength by $47 \%$ and $67 \%$ respectively [25]. Among various nanofillers, GO nanosheets with plentiful carboxylic and hydroxyl functional groups have been proved to have excellent antifouling properties [26, 27]. Moreover, the functional groups can provide opportunities to attach metal and metal oxide NPs in order to improve application performance in the antifouling field [28-31]. Kim et. al. have developed GO nanopaints that exhibit not only antibacterial properties in lab tests, but also antifouling properties in field tests [32]. Especially, Qin et al. have developed GO-based antifouling coating on sensor surfaces via layer-by-layer technique without compromising the sensor's performance [33]. The valuable antifouling property and adaptability on sensor surfaces makes GO a promising nanofiller in PDMS. In addition, metal oxide NPs like copper oxide $(\mathrm{CuO})$, zinc oxide $(\mathrm{ZnO})$ and titanium dioxide $\left(\mathrm{TiO}_{2}\right)$ have been used as nanofillers in order to enhance the antifouling property of the PDMS matrix [20, 22, 34-37]. Among these NPs, $\mathrm{ZnO}$ exhibits various advantages, such as low cost, environmental friendliness and optimal antifouling property owing to its highest hydrophobicity and surface roughness. The selective toxicity to bacteria and low toxicity to human cells of $\mathrm{ZnO}$ makes it a promising candidate in the antifouling field [36, 38, 39].

In light of these facts, $\mathrm{ZnO}-\mathrm{GO}$ nanocomposite has been prepared through the nucleation of $\mathrm{ZnO}$ NPs in the abundant functional groups of GO's surfaces [40]. With the incorporation of $\mathrm{ZnO}-\mathrm{GO}$ into PDMS, we have thus obtained a serious of PZGO nanocomposites with different mass ratio (from 0.05 to $2 \% \mathrm{wt} \%$ ) of $\mathrm{ZnO}-\mathrm{GO}$ to PDMS. In this work, PZGO nanocomposite has been developed with the aims of addressing short-term biofouling effect of sensors. Owing to the high surface roughness $(4.02 \mathrm{~nm})$ and hydrophobicity $\left(117^{\circ}\right)$ of the developed PZGO nanocomposite and antimicrobial property of $\mathrm{ZnO}$ NPs, PZGO nanocomposite delivered competitive antifouling performance with $97.6 \%$ fouling inhibition. The study provides an alternative for developing antifouling coatings to address sensor's durability problem caused by fouling. 


\section{Experimental}

\section{Materials}

Graphene oxide (GO) was purchased from Abalonyx Norway. Zinc acetate dehydrate $\left(\mathrm{Zn}\left(\mathrm{CH}_{3} \mathrm{COO}\right)_{2} \cdot 2 \mathrm{H}_{2-}\right.$ $\mathrm{O}, \geq 99.0 \%$ ), Lithium hydroxide monohydrate ( $\left.\mathrm{LiOH} \cdot \mathrm{H}_{2} \mathrm{O}, \geq 98.0 \%\right), \quad$ Tetrahydrofuran $\quad\left(\mathrm{C}_{4} \mathrm{H}_{8-}\right.$ $\mathrm{O}, \geq 99.9 \%)$, absolute alcohol $\left(\mathrm{CH}_{3} \mathrm{CH}_{2} \mathrm{OH}, 100 \%\right)$, n-hexane $\left(\mathrm{CH}_{3}\left(\mathrm{CH}_{2}\right)_{4} \mathrm{CH}_{3}, \geq 96.0 \%\right)$ glutaraldehyde solution (grade 1, 25\% in $\mathrm{H}_{2} \mathrm{O}$ ) and Sylgard(R) 184 were obtained from Sigma-Aldrich.

\section{Preparation of ZnO-GO Nanocomposite}

As shown in Scheme 1 (1), a facile one-pot reaction was used to synthesized $\mathrm{ZnO}-\mathrm{GO}$ composites [40]. In brief, $0.55 \mathrm{~g} \mathrm{Zn}\left(\mathrm{CH}_{3} \mathrm{COO}\right)_{2} \cdot 2 \mathrm{H}_{2} \mathrm{O}$ was added into $50.0 \mathrm{ml}$ absolute ethanol and dissolved by stirring under $80{ }^{\circ} \mathrm{C}$ for $15 \mathrm{~min}$, the obtained mixture was then cooled to $40{ }^{\circ} \mathrm{C}$. Subsequently, $0.2 \mathrm{~g}$ dissolved $\mathrm{LiOH} \cdot \mathrm{H}_{2} \mathrm{O}$ in $30 \mathrm{ml}$ alcohol and varying amounts of $2 \mathrm{mg} / \mathrm{ml} \mathrm{GO}$ aqueous solution were mixed with the above solution by stirring for $45 \mathrm{~min}$ under $40{ }^{\circ} \mathrm{C}$. After cooling and the addition of $100 \mathrm{ml}$ n-hexane, the final mixture was left overnight at $4{ }^{\circ} \mathrm{C}$ The resulting precipitates were then separated by washing with DI water and absolute ethanol for three times in turn under centrifugation. Finally, the $\mathrm{ZnO}-\mathrm{GO}$ nanocomposites were obtained by vacuum drying at $60{ }^{\circ} \mathrm{C}$ for $12 \mathrm{~h}$.

\section{Preparation of PDMS/ZnO-GO Surface}

Scheme 1 (2) represents the preparation of PZGO nanocomposites coating surface. $\mathrm{ZnO}-\mathrm{GO}$ was first dispersed in tetrahydrofuran (THF) by probe sonication for 20 min. The suspension was then added into PDMS (part A), followed by water bath sonication for $30 \mathrm{~min}$ and then mechanical stirring (1000 rpm) for $30 \mathrm{~min}$. Subsequently, part B (the ratio of part A to part B is 10:1) was mixed with above mixture through mechanical stirring until all THF solvent had completely evaporated. The obtained PZGO mixture was then placed in a vacuum desiccator and degassed to remove the air bubbles. Finally, the PZGO mixture was spin-coated (1000 rpm, $30 \mathrm{~s}$ ) on round cover slips which had been rinsed with water and ethanol to get a thin film on one side of each cover slip. Curing was carried out in an oven at $65{ }^{\circ} \mathrm{C}$ for $4 \mathrm{~h}$. Pristine PDMS surface worked as control sample. The control was made by mixing part A and part B in a ratio of 10:1, following the same procedures as the preparation of PZGO film. In this work, PZGO nanocomposites were named based on the mass ratio of $\mathrm{ZnO}-\mathrm{GO}$ to PDMS, as summarized in Table 1. For example, PZGO0.05 represents $0.05 \mathrm{wt} \%$ mass ratio of $\mathrm{ZnO}-\mathrm{GO}$ to PDMS.

\section{Characterization}

Fourier transform infrared (FTIR) spectra were recorded with a Bruker ALPHA FTIR spectrometer. X-ray diffraction (XRD) was obtained with DaVincil from $10^{\circ}$ to $80^{\circ}$. UV-visible absorption spectra were recorded by Cary 60 $\mathrm{UV}$-vis at the range of 200 to $500 \mathrm{~nm}$. Raman spectra were
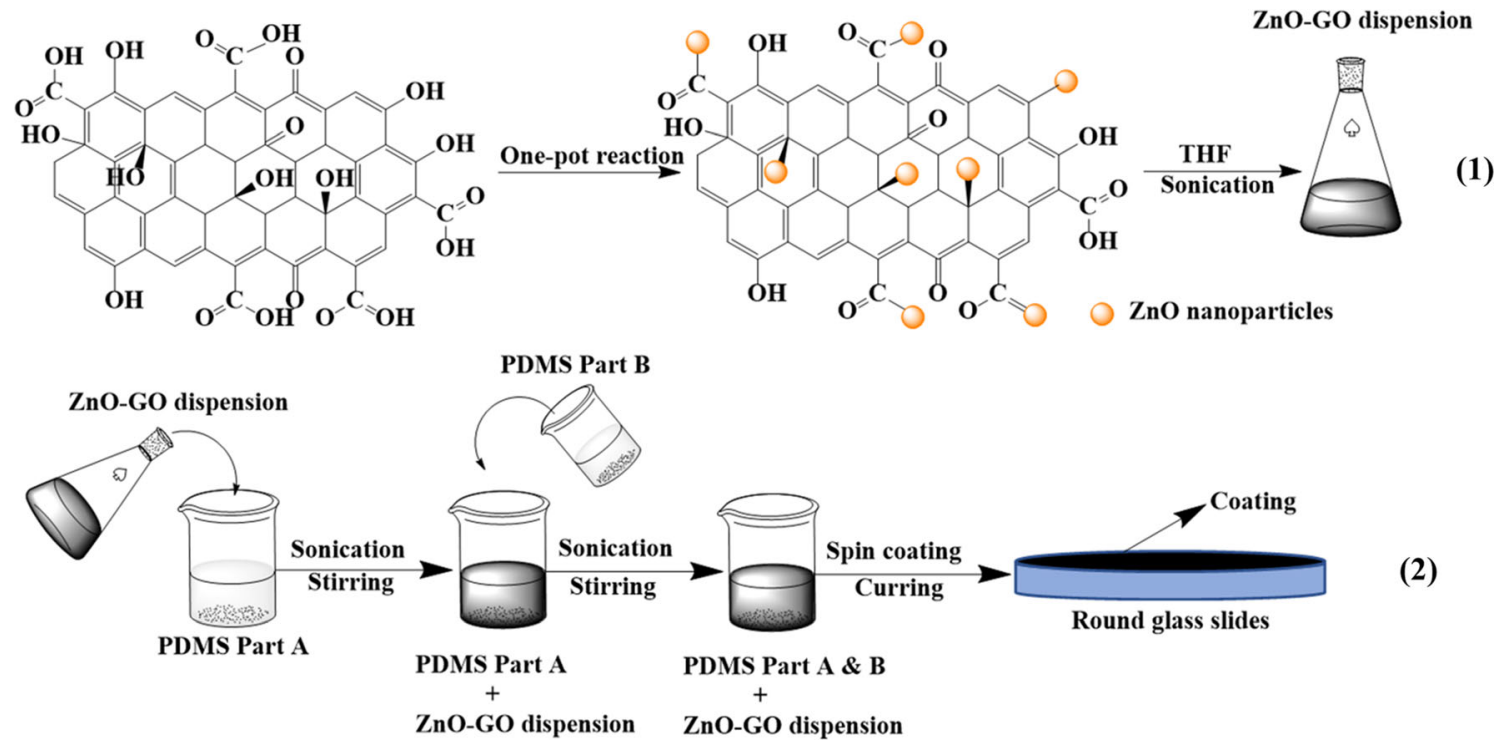

Scheme 1 (1) Preparation of ZnO-GO nanocomposites through a one-pot method. (2) Preparation of PZGO nanocomposite and spin coating the coating surface 
Table 1 Different mass ratios of $\mathrm{ZnO}-\mathrm{GO}$ to PDMS in the formation of PZGO

\begin{tabular}{lllll}
\hline Name & PZGO0.05 & PZGO0.1 & PZGO0.2 & PZGO2 \\
\hline ZnO-GO: PDMS & $0.5 \times 10^{-3}: 1$ & $1 \times 10^{-3}: 1$ & $2 \times 10^{-3}: 1$ & $20 \times 10^{-3}: 1$ \\
\hline
\end{tabular}

determined by InVia Reflex Spectrometer System with $532 \mathrm{~nm}$ laser excitation. Scanning electron microscopy (SEM) images were obtained by using FEI APREO, which is a Field Emission SEM. Transmission electron microscopy (TEM) images were obtained with in-lens cold field emission microscopy (Hitachi S-5500 S(T)EM) with an accelerating voltage at $30 \mathrm{kV}$. X-ray spectrometer (EDX) were investigated for element analysis of samples. Raman spectra were recorded by InVia Reflex Spectrometer System with $532 \mathrm{~nm}$ laser excitation. The surface property of the synthesized materials was analyzed with Atomic force Microscopy (AFM, Veeco Metrology). AFM was used to measure surface morphology and roughness under PeakForce Quantitative NanoMechanics mode. Contact angle and surface free energy (SFE) were measure with drop shape analyzer DSA25 (KRÜSS) by sessile drop technique. Static water contact angle measurements were performed with deionized water five times for each sample. Dynamic WCA including advancing contact angle $\left(\theta_{\text {adv }}\right)$ and receding contact angle $\left(\theta_{\text {rec }}\right)$ was measured with $25 \mu \mathrm{l}$ of deionized water being expanding and shrinking on the sample at a rate of $0.75 \mu \mathrm{l} / \mathrm{s}$. The difference between $\theta_{\mathrm{adv}}$ and $\theta_{\text {rec }}$ is referred as hysteresis. SFE was determined through measuring surface contact angles of pure water (polar liquid) and diiodomethane (disperse liquid). In this work, we Owens, Wendt, Rabel and Kaelble (OWRK model) $[41,42]$ was applied to calculate the SFE of PDMS and PZGO nanocomposite. This model was also used by our previous work to calculate SFE [43].

\section{Preparation of Bacterial Strains and Bacteria Adhesion Testing}

Synechococcus sp. Strain PCC 7002, a marine cyanobacterium, was inoculated and cultured in $40 \mathrm{~mL}$ batches of AA+-medium in $75 \mathrm{~cm}^{3}$ Nunc Easyflask culture flasks with filter caps. The cultures were grown overnight on a shaker at $30{ }^{\circ} \mathrm{C}$ and $30 \mu \mathrm{mol}$ photons $\mathrm{m}^{-2} \mathrm{~s}^{-1}$ to $\mathrm{OD}_{730}$ of roughly 0.4. AA+-medium is a derivative of $\mathrm{A}+$ [44], with the P1 trace metal solution replaced by a very similar 1000X BG-11 trace mineral solution [45].

The antibacterial activity of PDMS and PZGO was studied by testing against Synechococcus sp. Strain PCC 7002. In brief, the sterilized coverslips with PDMS and PZGO were placed into each well of 12-well plates. $5 \mathrm{ml}$ of Synechococcus sp. Strain PCC 7002 culture with $\mathrm{OD}_{730}$ of 0.4 was added into each well. The soaked coverslips in each well were incubated at $30{ }^{\circ} \mathrm{C}$. After incubation for
7 days, the unattached cells were removed from the coverslips by washing coverslips with $\mathrm{AA}+$ medium three times. Subsequently, the attached biofilm on coverslips was fixed with $2.5 \%$ glutaraldehyde for $2 \mathrm{~h}$ at $4{ }^{\circ} \mathrm{C}$ before measuring with confocal laser scanning microscopy (CLSM). In this work, coverage $(\%)$ was defined as the ratio of the adhesion area of the cells to the total area of surface. Five points were selected randomly for each sample, resulting in the statistical data. The standard Student t-test was used to compare the difference between control glass slides and modified glass slides.

\section{Preparation of Algal Cultures and Algal Adhesion Testing}

An axenic culture of the marine diatom $P$. tricornutum (Bacillariophyceae) clone Pt 1.8.6 (CCMP2561) obtained from the culture collection of the Provasoli-Guillard National Center for Culture of Marine Phytoplankton (Bigelow Laboratory for Ocean Sciences, USA) was used for the adhesion testing. P. tricornutum cultures were grown in $\mathrm{f} / 2$ medium made with $0.2 \mu \mathrm{m}$ filtered and autoclaved local seawater supplemented with $\mathrm{f} / 2$ vitamins and inorganic nutrients, filter sterilized and added after autoclaving (Guillard, 1975). Cultures were grown under a $16 \mathrm{~h}$ photoperiod at $22{ }^{\circ} \mathrm{C}, 35 \mu \mathrm{mol}$ photons $\mathrm{m}^{-2} \mathrm{~s}^{-1}$.

Coverslips with coatings of PDMS and PZGO were firstly sterilized with EtOH (10 s) and then placed into each well of 12 -well plates. $5 \mathrm{ml}$ of $P$. tricornutum with a cell concentration of $1 \times 10^{5}$ cells $/ \mathrm{ml}$ was added into individual wells and maintained at $22{ }^{\circ} \mathrm{C}$. After one week of incubation, coverslips were taken out from each well and washed with f/2 medium three times to remove loosely attached algae. Subsequently, the coverslips with attached algae was fixed with $2.5 \%$ glutaraldehyde for $2 \mathrm{~h}$ at $4{ }^{\circ} \mathrm{C}$, and then imaged with CLSM. Five random fields were imaged for each sample. The coverage (\%) of attached algae were quantified with ImageJ software through calculating CLSM images.

\section{Results and discussion}

\section{Structure and Morphology Analysis of ZnO-GO}

ZnO-GO nanocomposite was synthesized through a facile one-pot reaction. Figure 1A shows FTIR spectra of GO and $\mathrm{ZnO}-\mathrm{GO}$ nanocomposite. It can be seen from the spectrum 
Fig. 1 (A) FTIR spectra of GO and $\mathrm{ZnO}-\mathrm{GO}$ nanocomposite; (B) UV spectra of GO and $\mathrm{ZnO}-\mathrm{GO}$ nanocomposites; (C) XRD spectra of GO and $\mathrm{ZnO}-\mathrm{GO}$ nanocomposites
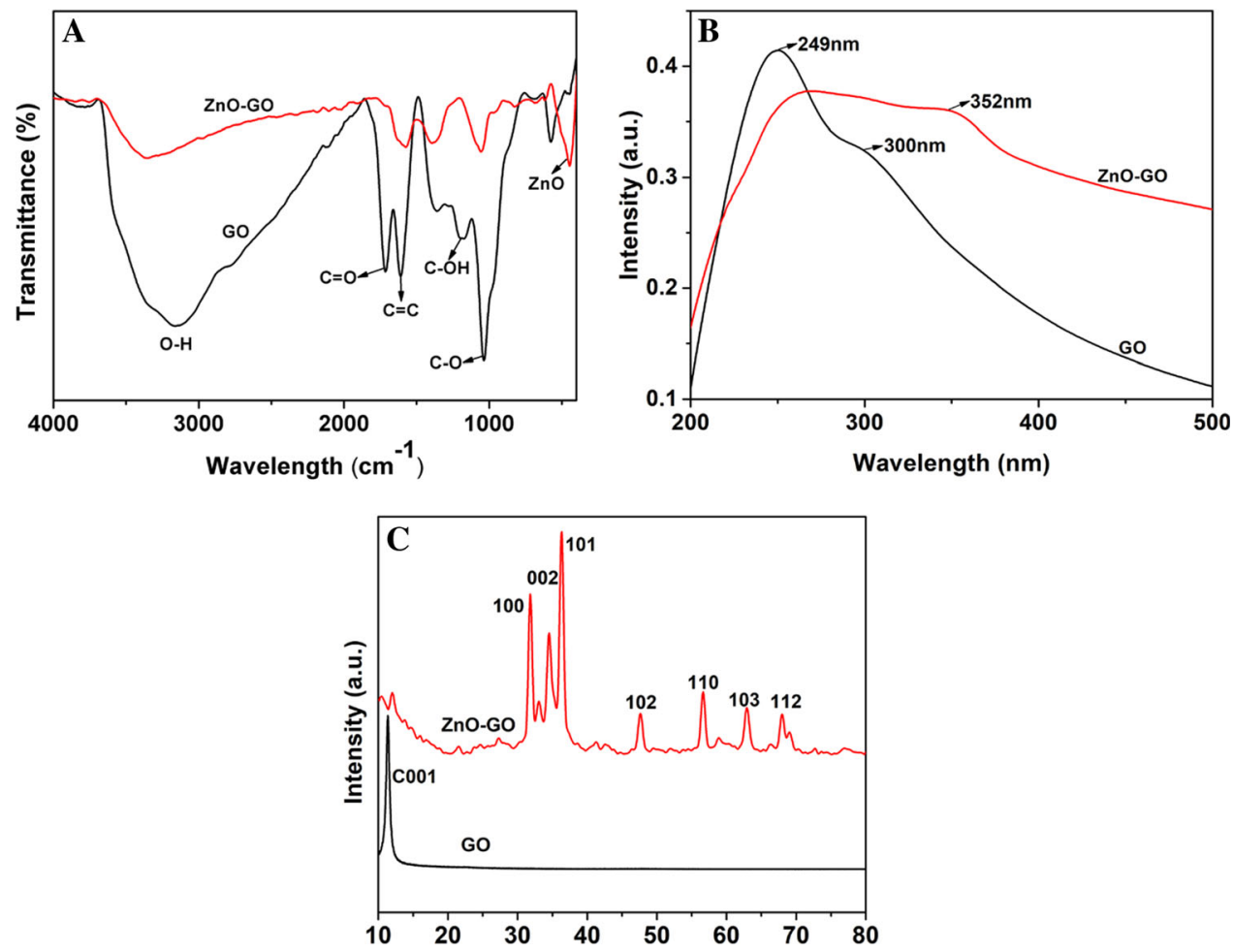

of GO that the peaks at around $3170,1712,1602,1187$ and $1035 \mathrm{~cm}^{-1}$ are assigned to $\mathrm{O}-\mathrm{H}$ stretching vibration, $\mathrm{C}=\mathrm{O}$ stretching vibration of $\mathrm{COOH}$ groups, skeletal vibration of unoxidized domains, $\mathrm{C}-\mathrm{OH}$ stretching vibrations and $\mathrm{C}-\mathrm{O}$ stretching vibrations, respectively [46]. In the spectrum of $\mathrm{ZnO}-\mathrm{GO}$, the weaker peaks of hydroxyl, carboxyl and epoxide groups indicate the reduction of GO. Furthermore, a new peak at around $447 \mathrm{~cm}^{-1}$ is attributed to $\mathrm{ZnO}$ which is in accordance with the UV spectrum that is discussed below [47]. As shown in the UV spectrum in Fig. 1B, GO exhibited a peak at around $249 \mathrm{~nm}$ and a shoulder at around $300 \mathrm{~nm}$ attributing to $\pi-\pi^{*}$ transition of $\mathrm{C}=\mathrm{C}$ bond and $\mathrm{n}-\pi^{*}$ in $\mathrm{C}=\mathrm{O}$ bond, respectively [48]. Besides, the peak at around $352 \mathrm{~nm}$ revealed the forming of $\mathrm{ZnO}$ NPs [49]. Moreover, the absorption peak at around $300 \mathrm{~nm}$ of $\mathrm{ZnO}-$ GO nanocomposites became unobvious, which further proved that GO has been reduced. All these indicators from the UV spectra are in accordance with FTIR spectra. Meanwhile, it is clear to see the weak peak at around $2 \theta=11.3^{\circ}$, from Fig. 1C, assigning to reduced GO, and the new appeared peaks at around $2 \theta=31.8^{\circ}, 34.5^{\circ}, 36.3^{\circ}$, $47.6^{\circ}, 56.7^{\circ}, 62.9^{\circ}, 68.0^{\circ}$ attributing to the decorating of ZnO NPs (JCPDS No. 36-1451) on GO surface. Overall, the FTIR, UV and XRD spectra of GO and $\mathrm{ZnO}-\mathrm{GO}$ nanocomposites are consistent.

The morphology of $\mathrm{ZnO}-\mathrm{GO}$ nanocomposites was examined through SEM and TEM. As shown in Fig. 2A, some wrinkles were observed on GO, which provided a large surface for ZnO NPs to attach and were important for preventing aggregation of $\mathrm{ZnO}$ NPs. To estimate the chemical compositions of $\mathrm{ZnO}-\mathrm{GO}$ nanocomposite, EDX measurement was performed. Figure 2D shows presence of $\mathrm{Zn}, \mathrm{O}$ and $\mathrm{C}$ in sample. Si comes from the sample substrate where the sample was prepared. The SEM and TEM images and EDX sprectrum of $\mathrm{ZnO}-\mathrm{GO}$ demonstrated $\mathrm{ZnO}$ NPs were deposited on the surface of GO, and this is consistent with FTIR, UV and XRD results.

\section{Structure and Surface Properties of PDMS and PZGO Nanocomposites}

Raman spectra of PDMS and PZGO nanocomposites are presented in Fig. 3. It was noticed that the peak at around 487,619 and $707 \mathrm{~cm}^{-1}$ are attributed to symmetric Si-O$\mathrm{Si} \mathrm{cm}{ }^{-1}$, rocking of $\mathrm{Si}-\mathrm{CH}_{3}$ and symmetric stretching of $\mathrm{Si}-\mathrm{C}$, respectively. The $\mathrm{Si}-\mathrm{CH}_{3}$ symmetric and asymmetric stretching bands where at 2904 and $2964 \mathrm{~cm}^{-1}$. Regarding the PZGO nanocomposites, no markedly difference was noticed in the spectra of PZGO 0.05 and PZGO0 due to the low content of $\mathrm{ZnO}-\mathrm{GO}$. With the increased amount of $\mathrm{ZnO}-\mathrm{GO}$, two new peaks at around 1348 and $1597 \mathrm{~cm}^{-1}$, as shown in the insertion Fig. 3, were observed. These two peaks correspond to the dominant peaks of GO, which indicated the presence of $\mathrm{ZnO}-\mathrm{GO}$ nanocomposites.

Figure 4 shows SEM images of pure PDMS and PZGO nanocomposites. PDMS displays smooth and featureless surface property, as shown in Fig. 4A. $\mathrm{ZnO}-\mathrm{GO}$ nanofillers can be clearly observed from Fig. 4B-E. The SEM images 
Fig. 2 SEM pictures of A GO, B $\mathrm{ZnO}-\mathrm{GO}$, C TEM picture of $\mathrm{ZnO}-\mathrm{GO}$ nanocomposite,

D EDX spectrum of $\mathrm{ZnO}-\mathrm{GO}$
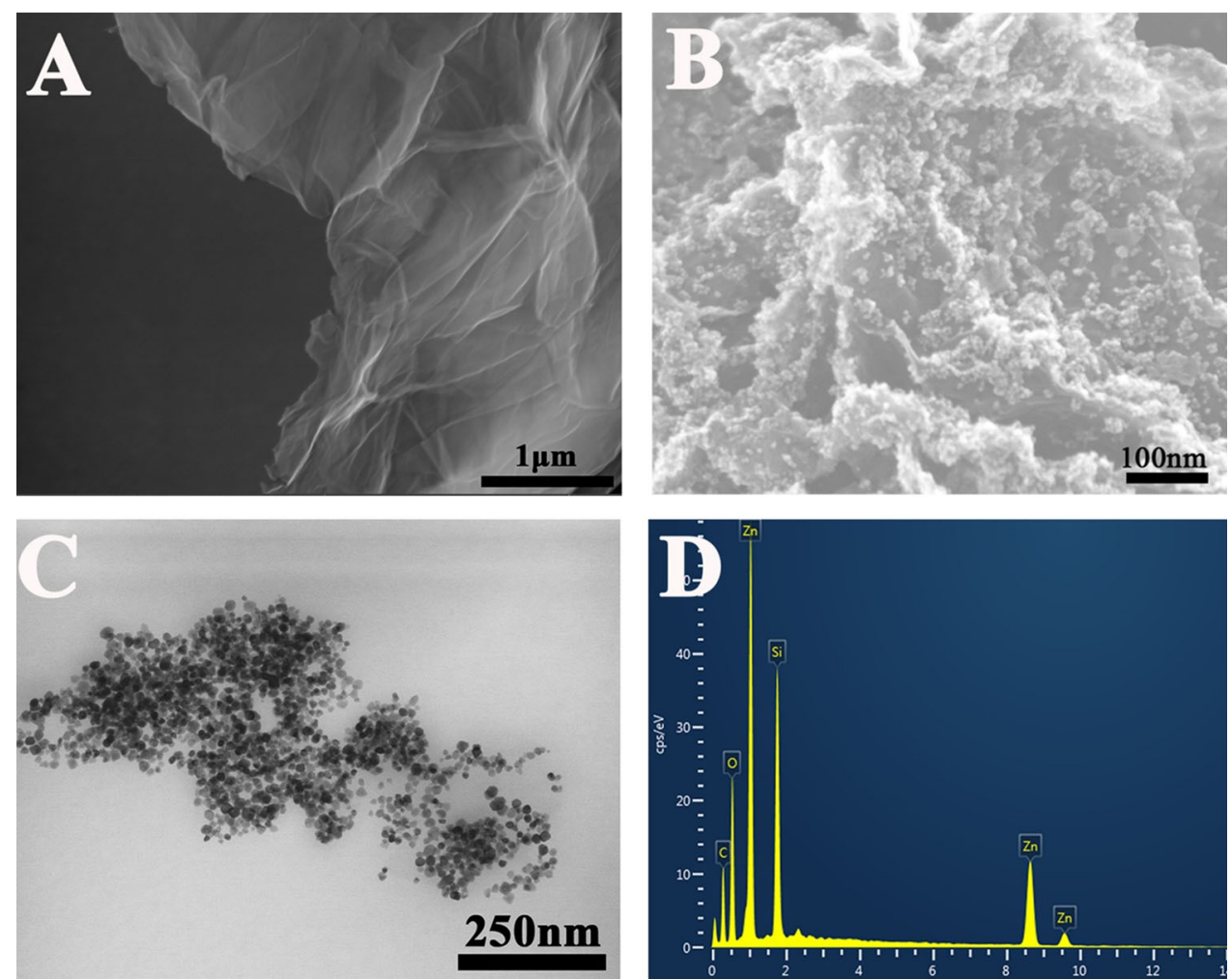

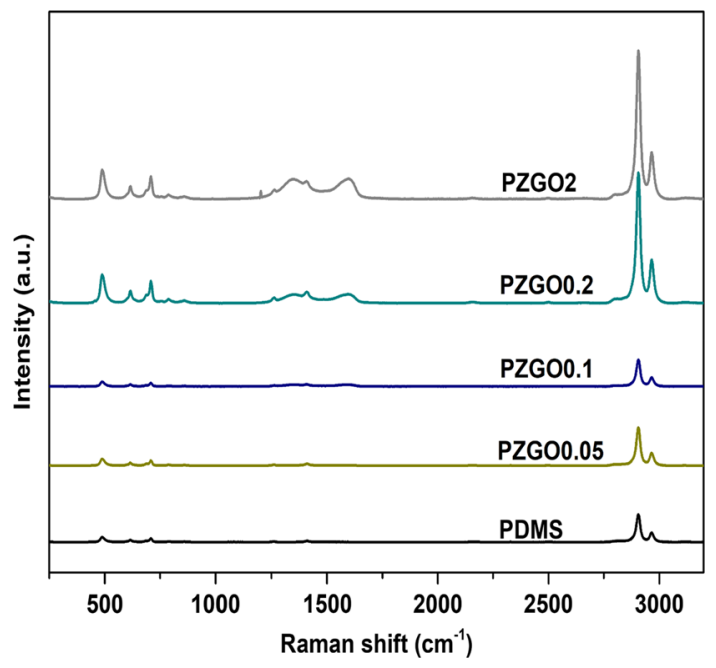

Fig. 3 Raman spectra of PDMS and PZGO nanocomposites with different mass ratios of $\mathrm{ZnO}-\mathrm{GO}$ to PDMS

of PZGO nanocomposites were studied to determine the effect of the distribution of $\mathrm{ZnO}-\mathrm{GO}$ on the surface properties of PZGO nanocomposites. PZGO0.05, PZGO0.1 and PZGO0.2 exhibited well-dispersion and no aggregation characteristics. The good dispersion of $\mathrm{ZnO}-\mathrm{GO}$ nanofillers in PDMS matrix resulted in a homogenous surface which could reduce fouling settlement. Conversely, the increased loading of nanofillers after PZG0.2 caused clustering and accumulation of $\mathrm{ZnO}-\mathrm{GO}$ in PDMS matrix.
Nanofillers' aggregation decreased surface homogeneity, thus facilitating fouling settlements.

PDMS and PZGO nanocomposites' topographies were investigated via AFM as shown in Fig. 5. Plain PDMS showed a surface roughness (Ra) of $2.53 \mathrm{~nm}$. PZGO nanocomposites exhibited a progressive increase of $\mathrm{Ra}$ with raised incorporation of $\mathrm{ZnO}-\mathrm{GO}$ nanofillers. It showed the rough surface peaked at PZGO0.2 with the value of $4.02 \mathrm{~nm}$, resulting from the good distribution of $\mathrm{ZnO}-\mathrm{GO}$ nanofillers. However, the continuous boost of $\mathrm{ZnO}-\mathrm{GO}$ nanofillers led to inhomogeneous surface due to aggregation, which in turn diminished $\mathrm{Ra}$ of $\mathrm{PZGO} 2$ to $3.28 \mathrm{~nm}$.

Wetting properties and SFE of PZGO nanocomposites filled with different amount of $\mathrm{ZnO}-\mathrm{GO}$ nanofillers compared to plain PDMS are shown in Fig. 6. The pure PDMS showed a contact angle of $107^{\circ}$ and so exhibited hydrophobic behavior. There was a gradually increase of WCA with raised loading of $\mathrm{ZnO}-\mathrm{GO}$ nanofillers into PDMS. WCA raised up to $117^{\circ}$ by the introduction of 0.2 wt $\%$ nanofiller indicating better hydrophobicity. But higher amount of nanofillers after PZGO0.2 does not lead to a continuous raising of hydrophobicity and Ra due to inhomogeneous surface caused by aggregation of $\mathrm{ZnO}-\mathrm{GO}$. In addition, with good dispersibility of $\mathrm{ZnO}-\mathrm{GO}$, the increased content of $\mathrm{ZnO}-\mathrm{GO}$ nanofillers reduced the SFE from $20.02 \mathrm{mN} / \mathrm{m}$ (PDMS) to $9.06 \mathrm{mN} / \mathrm{m}$ (PZGO0.2), whereas the cluster of $\mathrm{ZnO}-\mathrm{GO}$ nanofillers increased SFE. 
Fig. 4 SEM images of A PDMS, B PZGO0.05,

C PZGO0.1, D PZGO0.2, E PZGO2

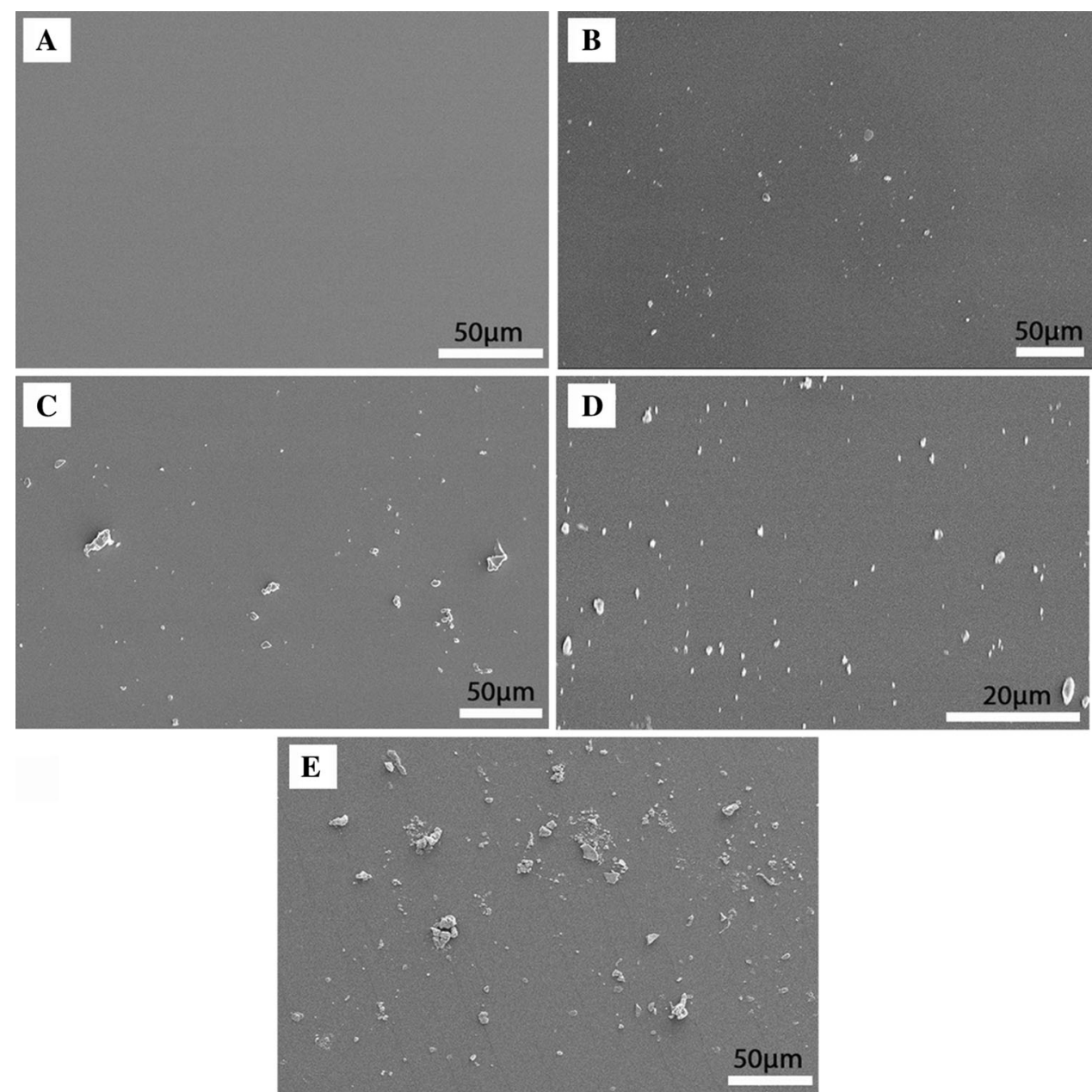

The results of Ra (Fig. 5F), WCA and SFE reflect that, with good distribution, raised content of $\mathrm{ZnO}-\mathrm{GO}$ induces high Ra, high WCA and low SFE, which benefit for antifouling property. On the other hand, the cluster of ZnO-GO has negative impact on antifouling property due to low Ra, low WCA and high SFE. Furthermore, as shown in Fig. 6B, the incorporation of $\mathrm{ZnO}-\mathrm{GO}$ nanofiller does not have an obvious impact on neither $\theta_{\text {adv }}$ nor $\theta_{\text {rec. }}$. However, $\triangle \theta$ increased from $31^{\circ}$ to $55^{\circ}$ with the amount of $\mathrm{ZnO}-\mathrm{GO}$ increasing from $0.2 \mathrm{wt} . \%$ to $2 \mathrm{wt} . \%$, resulting from aggregation of $\mathrm{ZnO}-\mathrm{GO}$.

\section{Antifouling tests}

\section{Bacteria}

Visual confirmation of antifouling effect against Synechoccocus sp 7002 of pure PDMS and PZGO nanocomposites was investigated by CLSM. As shown in Fig. 7A, the biofilm fully covered the surface of pure PDMS after 7 days incubation. As observed from Fig. 7B to Fig. 7E, the incorporation of $\mathrm{ZnO}-\mathrm{GO}$ nanofillers can decrease the attachment of Synechoccocus sp 7002 significantly. Significantly lower green fluorescence was observed on the surface of PZGO0.2 and PZGO2 compared to pure PDMS. Statistical analysis of Synechoccocus sp 7002 fouling was obtained through Image J. As shown in Fig. 7F, the increased amount of $\mathrm{ZnO}-\mathrm{GO}$ nanofillers resulted in gradually reduced biofilm attachment until PZGO0.2, which indicates improved antifouling property. On the other hand, the continuous increased loading of $\mathrm{ZnO}-\mathrm{GO}$ after PZGO0.2 caused a slight increase of biofilm coverage due to serious aggregation of $\mathrm{ZnO}-\mathrm{GO}$. Aggregations results in nonhomogeneous topology and in turn leads to more fouling attachment on the clustered surface. A good dispersion of nanofillers produces rougher surface, higher hydrophobicity and lower biofilm attachment. Notably, PZGO0.2 presents the best antifouling property with only 8.5\% biofilm coverage, while pure PDMS shows 55.2\% biofilm coverage. Also, PZGO0.2 displays the highest Ra of $4.02 \mathrm{~nm}$ and the best hydrophobicity of $117^{\circ}$ based on surface analysis. Above all, these results imply that distribution of nanofillers is crucial to surface topology and fouling inhibition. It is reported that the increased 

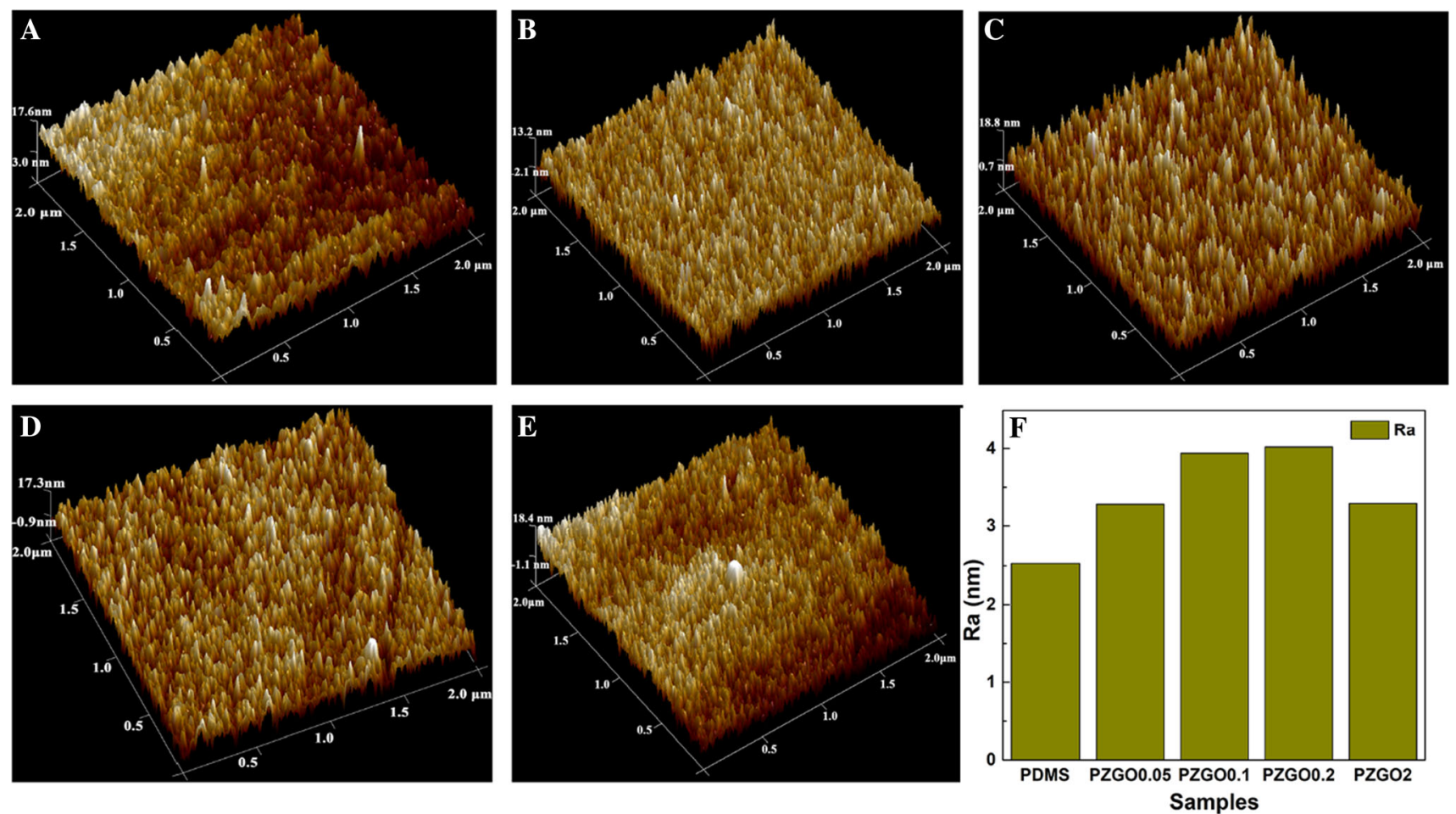

Fig. 5 3D AFM images of A PDMS, B PZGO0.05, C PZGO0.1, D PZGO0.2, E PZGO2
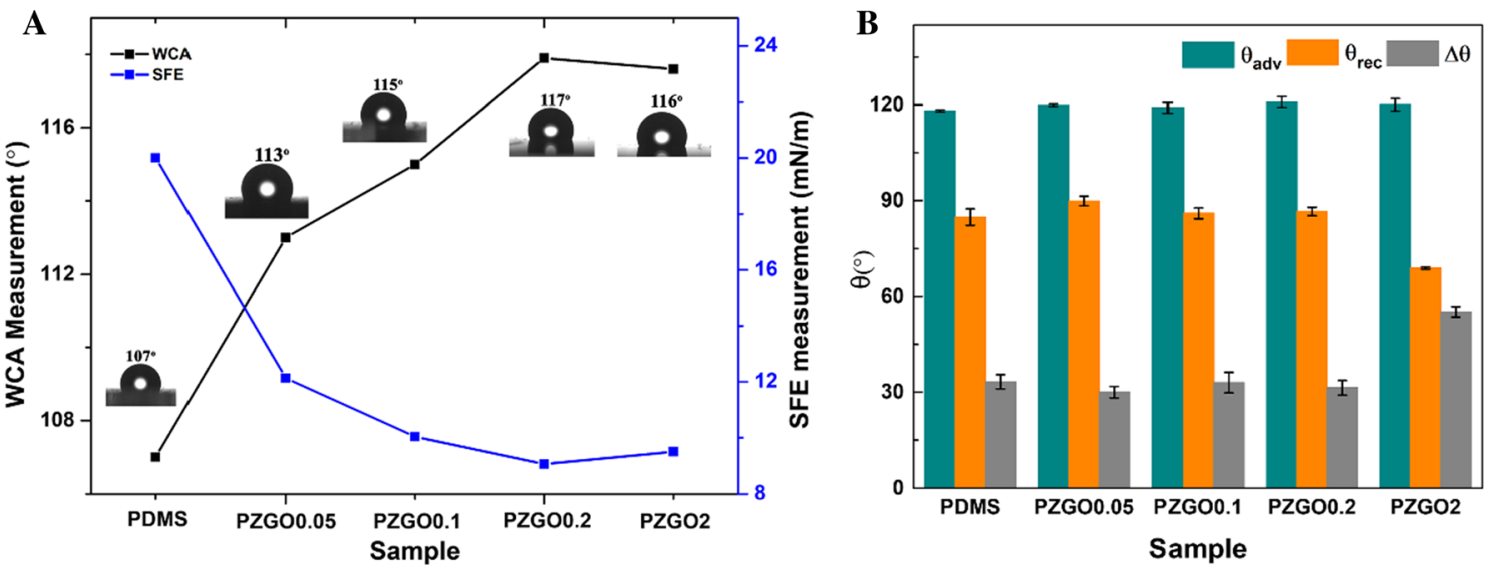

Fig. 6 A Comparison of WCA and SFE of PDMS and PZGO nanocomposites; B Dynamic water contact angle of PDMS and PZGO nanocomposites

nanoscale surface roughness and hydrophobicity can lead to good antifouling property. The reason for this is that the nanoscale surface roughness can trap air between surface interstices, so that the rough surface is equivalent to a composite surface of vapor and solid. Such enhanced rough surface (Scheme 2) caused by the incorporation of $\mathrm{ZnO}-$ GO nanofillers can prevent water from penetrating into interstices' groves, which consequently leads to increased hydrophobic property $[50,51]$. Also, this phenomenon can afford increased antifouling property caused by declined surface-fouling bond [51, 52]. In addition, antimicrobial property of $\mathrm{ZnO}-\mathrm{GO}$ nanofillers also contributes to the decreased fouling attachment.

\section{Algae}

To investigate antifouling property against microalgae, round glass slides with pure PDMS and PZGO coating surfaces were immersed in $P$. tricornutum suspensions for 7 days. P. tricornutum is a typical biofouling organism because it participates in early stage of biofouling [53]. The CLSM images clearly indicates that good dispersion of 

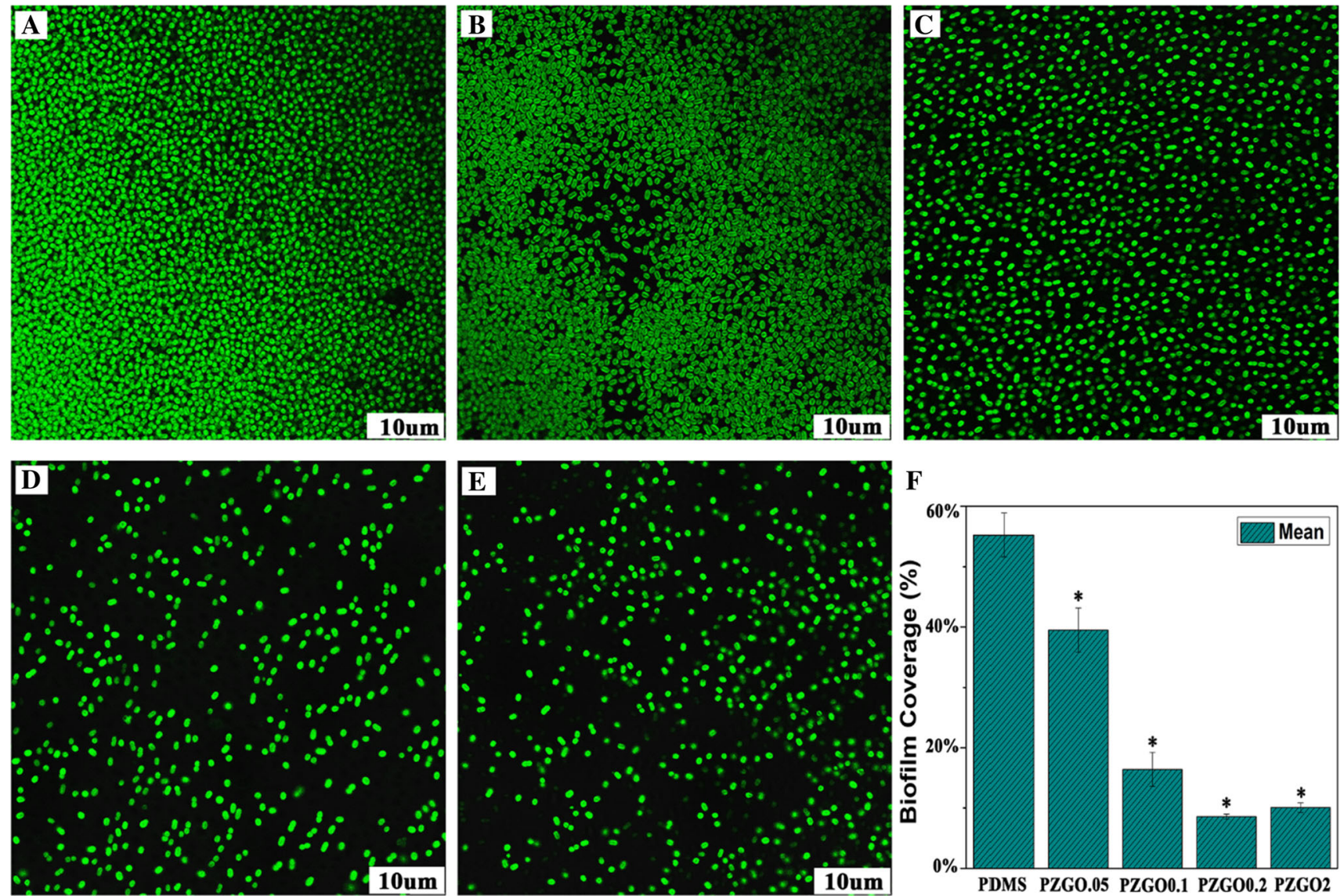

Fig. 7 CLSM images showing adhesion of Synechoccocus sp 7002 A PDMS, B PZGO0.05, C PZGO0.1, D PZGO0.2, E PZGO2; F, Statistic biofilm coverage (adhesion ratio) of Synechoccocus sp 7002, error bars are shown as $\pm \mathrm{SD}(\mathrm{n}=5)$. $* \mathrm{P}<0.05$

Scheme 2 Hydrophobic behavior of PZGO nanocomposite that prevent fouling adhesion

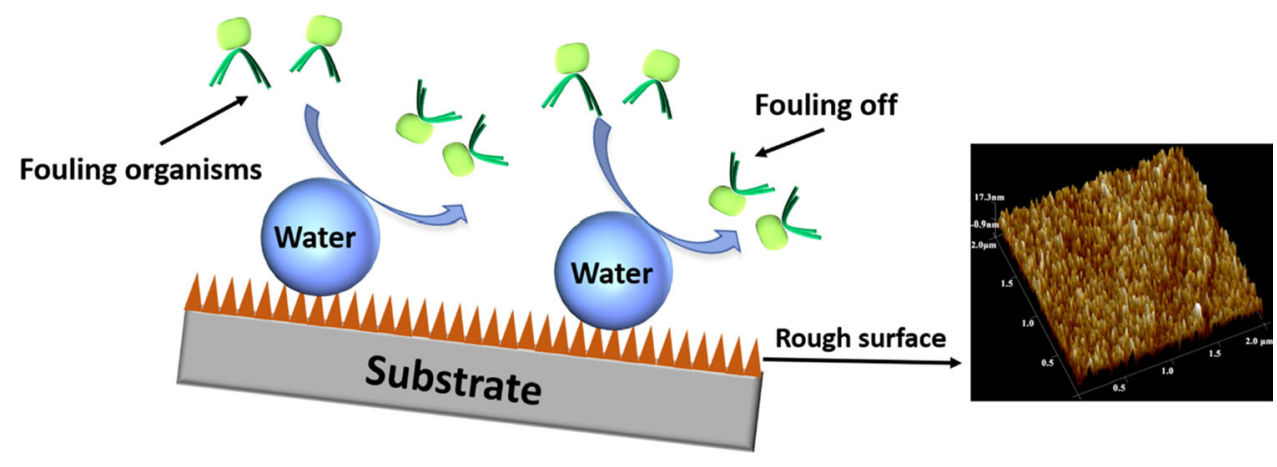

ZnO-GO (Fig. 8B-D) provided less biofilm attachment, while aggregation of $\mathrm{ZnO}-\mathrm{GO}$ (Fig. 8E) offered more serious fouling surface. This is because good dispersion of ZnO-GO brings about higher Ra and hydrophobicity, which are beneficial to antifouling property. Unlike the antifouling test with Synechoccocus sp 7002 where PZGO0.2 displayed the best antifouling property, PZGO0.1 resulted in the lowest biofilm coverage when performing antifouling tests with $P$. tricornutum. The biofilm coverage dropped from $43.7 \%$ when cells were grown on pure PDMS to $2.4 \%$ when the surface was coated with PZGO0.1
(Fig. 8F). It has been reported that the toxicity of ZnO NPs on algae is ascribed to the interaction between algae and $\mathrm{ZnO}$ NPs [54]. As for P. tricornutum, Cai et al. demonstrated that ZnO NPs can't attach on its surface and proved its resistance to the toxicity of $\mathrm{ZnO}$ NPs. Because the slender shape and surface charge of $P$. tricornutum can decrease the interaction with ZnO NPs and lead to low toxicity of $\mathrm{ZnO}$ NPs on P. tricornutum [55]. Therefore, it is hypothesized that the antifouling property of PZGO against $P$. tricornutum mostly comes from homogeneous topology, while antimicrobial effect of $\mathrm{ZnO}-\mathrm{GO}$ only contributes a 

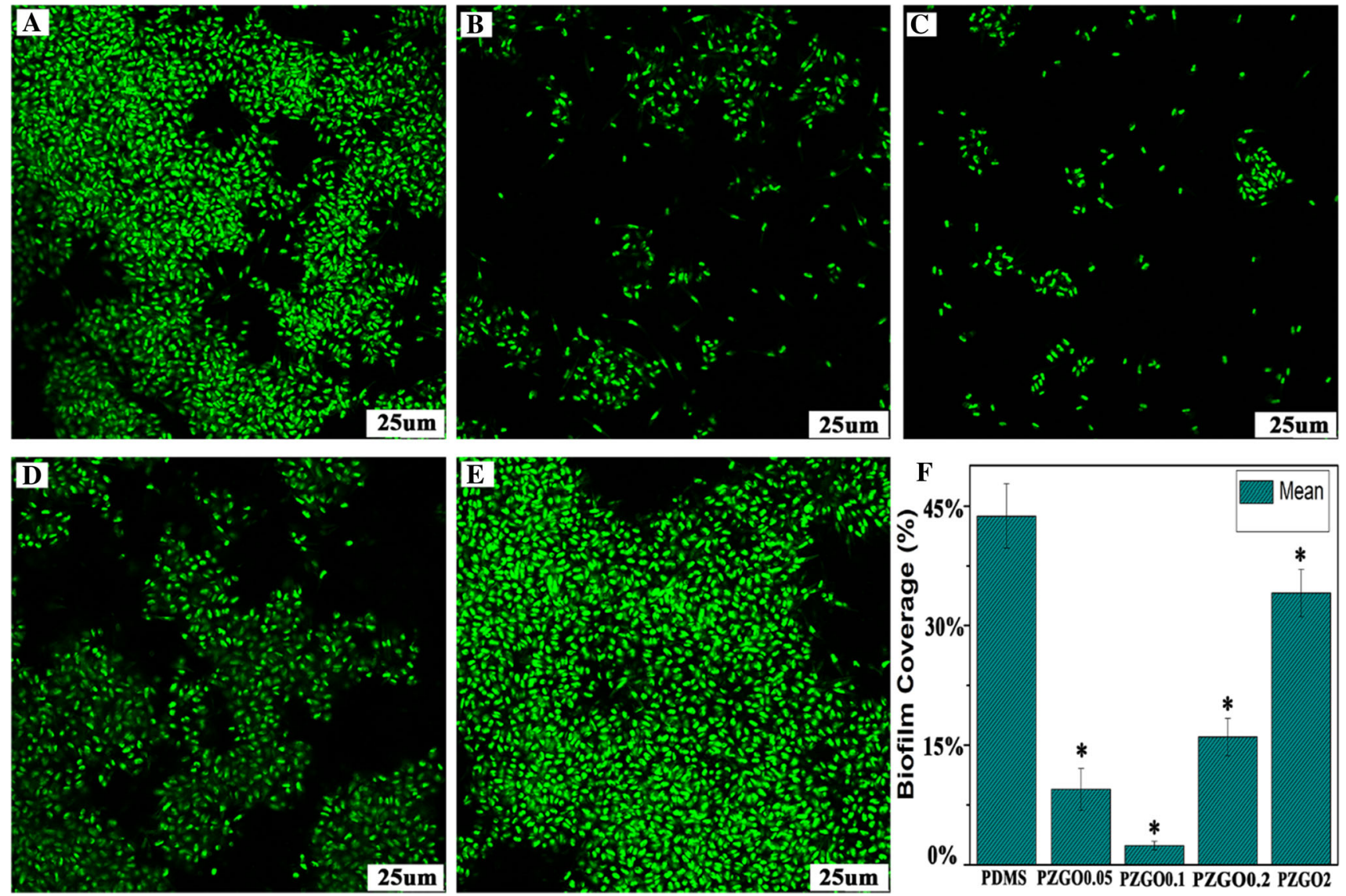

Fig. 8 CLSM images showing adhesion of $P$. tricornutum A PDMS, B PZGO0.05, C PZGO0.1, D PZGO0.2, E PZGO2; F, Statistic biofilm coverage (adhesion ratio) of $P$. tricornutum, error bars are shown as $\pm \mathrm{SD}(\mathrm{n}=5)$. $* \mathrm{P}<0.05$

little. Thus, the increased loading of $\mathrm{ZnO}-\mathrm{GO}$ from PZGO0.1 to PZGO0.2 does not cause a raised biofilm inhibition, because PZGO 0.1 and PZGO0.2 shows similar topology. But we're still unsure why the antifouling property of PZGO0.2 against $P$. tricornutum gets worse compared to PGZO0.1.

\section{Conclusion}

It is still a major challenge to improve the durability relating to biofouling resistance for sensors. In order to address the issue, a series of PZGO nanocomposites with different amount of $\mathrm{ZnO}-\mathrm{GO}$ nanofillers were spin coated on round glass slides for antifouling tests with two microorganisms. The higher hydrophobic property and Ra of the synthesized PZGO nanocomposites were beneficial from the good dispersion of $\mathrm{ZnO}-\mathrm{GO}$ nanofillers in PDMS, thus resulting in good antifouling property. The antifouling test proved that the PZGO nanocomposites showed superior antifouling property, but the optimal mass ratio of ZnO-GO to PDMS differed between Synechoccocus sp 7002 and P. tricornutum. PZGO0.2 showed the lowest biofilm coverage of $8.5 \%$ in the antifouling test against Synechoccocus sp 7002, while PZGO0.1 displayed the best antifouling property against $P$. tricornutum with $2.4 \%$ biofilm attachment. We believe that PZGO nanocomposite can provide a potential possibility for developing antifouling coating for sensor.

Acknowledgements The experimental work was undertaken in the NTNU NanoLab with a grant from NorFab [Project Number: 245963]. The Research Council of Norway is acknowledged for the support to the SENSOR Project [Grant Number: 237856].

Funding Open access funding provided by NTNU Norwegian University of Science and Technology (incl St. Olavs Hospital - Trondheim University Hospital).

Open Access This article is licensed under a Creative Commons Attribution 4.0 International License, which permits use, sharing, adaptation, distribution and reproduction in any medium or format, as long as you give appropriate credit to the original author(s) and the source, provide a link to the Creative Commons licence, and indicate if changes were made. The images or other third party material in this article are included in the article's Creative Commons licence, unless indicated otherwise in a credit line to the material. If material is not included in the article's Creative Commons licence and your intended use is not permitted by statutory regulation or exceeds the permitted use, you will need to obtain permission directly from the copyright 
holder. To view a copy of this licence, visit http://creativecommons. org/licenses/by/4.0/.

\section{References}

1. D. L. Schmidt, R. F. Brady, K. Lam, D. C. Schmidt, and M. K. Chaudhury (2004). Langmuir 20, 2830.

2. L. L. Xue, X. L. Lu, H. Wei, P. Long, J. Xu, and Y. F. Zheng (2014). J. Colloid Interface Sci. 421, 178.

3. O. Andersson, T. Ekblad, N. Aldred, A. S. Clare, and B. Liedberg (2009). Biointerphases 4, 65.

4. M. Cuartero, N. Pankratova, T. Cherubini, G. A. Crespo, F. Massa, F. Confalonieri, and E. Bakker (2017). Environ. Sci. Technol. Lett. 4, 410.

5. Z. A. Wang, F. N. Sonnichsen, A. M. Bradley, et al. (2015). Environ. Sci. Technol. 49, 4441.

6. T. Biard, L. Stemmann, M. Picheral, et al. (2016). Nature 532, 504.

7. L. Delauney, C. Compere, and M. Lehaitre (2010). Ocean Sci. 6 , 503.

8. I. Fitridge, T. Dempster, J. Guenther, and R. de Nys (2012). Biofouling 28, 649.

9. R. A. Braithwaite, M. C. C. Carrascosa, and L. A. McEvoy (2007). Aquaculture 262, 219.

10. K. H. Chae, Y. M. Jang, Y. H. Kim, O. J. Sohn, and J. I. Rhee (2007). Sens. Actuators B 124, 153.

11. P. H. Lin and B. R. Li (2020). Analyst 145, 1110.

12. A. Terlizzi, S. Fraschetti, P. Gianguzza, M. Faimali, and F. Boero (2001). Aquat. Conserv. 11, 311.

13. E. Yilgör and I. Yilgör (2014). Prog. Polym. Sci. 39, 1165.

14. C. J. Kavanagh, R. D. Quinn, and G. W. Swain (2005). J. Adhes. 81, 843.

15. P. J. Molino, E. Campbell, and R. Wetherbee (2009). Biofouling 25, 685 .

16. A. G. Nurioglu and A. C. C. Esteves (2015). J. Mater. Chem. B 3, 6547.

17. M. S. Selim, S. A. El-Safty, and M. A. Shenashen, Superhydrophobic Polymer Coatings (Elsevier, New York, 2019).

18. A. M. Elseman, M. S. Selim, L. Luo, et al. (2019). ChemSusChem. 12, 3808.

19. X. Li, M. A. Shenashen, X. Wang, A. Ito, A. Taniguchi, and S. A. Ei-Safty (2017). Sci. Rep. 7, 1.

20. M. S. Selim, S. A. El-Safty, A. Abbas, and M. A. Shenashen (2021). Colloids Surf A 611, 125793.

21. M. S. Selim, H. Yang, S. A. El-Safty, N. A. Fatthallah, M. A. Shenashen, F. Q. Wang, and Y. Huang (2019). Colloids Surf. A 570, 518.

22. M. S. Selim, M. A. Shenashen, A. Elmarakbi, N. A. Fatthallah, S.-I. Hasegawa, and S. A. El-Safty (2017). Chem. Eng. J. 320, 653.

23. A. Beigbeder, R. Mincheva, M. E. Pettitt, M. E. Callow, J. A. Callow, M. Claes, and P. Dubois (2010). J. Nanosci. Nanotechnol. 10, 2972.

24. A. Beigbeder, P. Degee, S. L. Conlan, et al. (2008). Biofouling 24, 291.

25. F. Irani, A. Jannesari, and S. Bastani (2013). Prog. Org. Coat. 76, 375.
26. I. N. Kholmanov, M. D. Stoller, J. Edgeworth, et al. (2012). Acs Nano 6, 5157.

27. O. Akhavan and E. Ghaderi (2010). Acs Nano 4, 5731.

28. Y. T. Chung, E. Mahmoudi, A. W. Mohammad, A. Benamor, D. Johnson, and N. Hilal (2017). Desalination 402, 123.

29. Q. Bao, D. Zhang, and P. Qi (2011). J. Colloid Interfaces Sci. 360, 463.

30. G. Williams, B. Seger, and P. V. Kamat (2008). Acs Nano. 2, 1487.

31. C. Xu, X. Wang, and J. W. Zhu (2008). J. Phys. Chem. C 112, 19841.

32. K. Krishnamoorthy, K. Jeyasubramanian, M. Premanathan, G. Subbiah, H. S. Shin, and S. J. Kim (2014). Carbon 72, 328.

33. T. J. Jiang, L. B. Qi, and W. Qin (2019). Anal. Chem. 91, 13268.

34. G. G. Sankar, S. Sathya, P. S. Murthy, A. Das, R. Pandiyan, V. P. Venugopalan, and M. Doble (2015). Int. Biodeterior. Biodegrad. 104, 307.

35. J. L. Yang, Y. F. Li, X. P. Guo, et al. (2016). Biofouling 32, 763.

36. C. Carl, A. J. Poole, B. A. Sexton, et al. (2012). Biofouling 28, 175.

37. M. S. Selim, S. A. El-Safty, A. M. Azzam, M. A. Shenashen, M. A. El-Sockary, and O. M. Abo Elenien (2019). ChemistrySelect 4, 3395.

38. R. F. Brady and I. L. Singer (2000). Biofouling 15, 73.

39. P. M. Sivakumar, S. Balaji, V. Prabhawathi, R. Neelakandan, P. T. Manoharan, and M. Doble (2010). Carbohydr. Polym. 79, 717.

40. Y. W. Wang, A. N. Cao, Y. Jiang, I. Zhang, J. H. Liu, Y. F. Liu, and H. F. Wang (2014). ACS Appl. Mater. Interfaces 6, 2791.

41. D. H. Kaelble (1970). J. Adhes. 2, 66-000.

42. D. K. Owens and R. C. Wendt (1969). J. Appl. Polym. Sci. 13, 1741-2000.

43. X. Zhang and Ø. Mikkelsen (2021). J. Clust. Sci. 13, 1741.

44. M. Ludwig and D. A. Bryant (2011). Front Microbiol. https://doi. org/10.3389/fmicb.2011.00041.

45. J. J. Eaton-Rye (2011). Methods Mol. Biol. 684, 295.

46. E. Y. Choi, T. H. Han, J. H. Hong, J. E. Kim, S. H. Lee, H. W. Kim, and S. O. Kim (2010). J. Mater. Chem. 20, 1907.

47. A. K. Zak, W. H. Abd Majid, M. Darroudi, and R. Yousefi (2011). Mater. Lett. 65, 70.

48. J. Q. Tian, S. Liu, H. Y. Li, et al. (2012). Rsc Adv. 2, 1318.

49. M. Azarang, A. Shuhaimi, R. Yousefi, and M. Sookhakian (2014). J. Appl. Phys. 116, 084307.

50. Y. B. Deng, D. Mager, Y. Bai, et al. (2018). Comput. Method Appl. M 341, 113.

51. M. S. Selim, H. Yang, F. Q. Wang, N. A. Fatthallah, Y. Huang, and S. Kuga (2019). Appl. Surf. Sci. 466, 40.

52. A. Shastry, M. J. Case, and K. F. Bohringer (2006). Langmuir 22, 6161.

53. X. Y. He, L. Abdoli, and H. Li (2018). Colloid Surf. B 162, 220.

54. J. J. Li, S. Schiavo, G. Rametta, M. L. Miglietta, V. La Ferrara, C. W. Wu, and S. Manzo (2017). Environ. Sci. Pollut. R 24, 6543.

55. C. Zhang, X. H. Chen, J. T. Wang, and L. J. Tan (2018). Environ. Eng. Sci. 35, 785.

Publisher's Note Springer Nature remains neutral with regard to jurisdictional claims in published maps and institutional affiliations. 